\title{
Study on the independent effect of thyroid hormone based on uric acid level on NAFLD
}

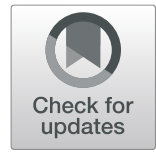

\author{
Guanqun Chao ${ }^{*}$ and Liying Chen
}

\begin{abstract}
Objective: This study aims to explain the correlation among non-alcoholic fatty liver disease (NAFLD), hyperuricemia, and thyroid function and to find independent risk factors for each other.

Methods: Data were obtained from subjects who underwent health examination in the Health Promotion Centre of Sir Run Run Shaw Hospital of Zhejiang University from January 2017 to February 2019. The diagnosis of NAFLD was according to the clinical diagnosis of the guidelines. Serum uric acid (SUA) $>360 \mu \mathrm{mol} / \mathrm{L}$ (female) and SUA > $420 \mu \mathrm{mol} / \mathrm{L}$ (male) were enrolled in the hyperuricemia group. R software was used for statistical analysis.
\end{abstract}

Results: 55,449 subjects were included in the analysis. $34.27 \%$ of patients were classified as NAFLD group ( $N=$ 19004), and $65.73 \%$ of patients were classified as non-NAFLD group ( $N=36445)$. The levels of gender ratio, age, $B M I$, waist circumference, systolic blood pressure (SBP), diastolic blood pressure (DBP), fasting blood glucose (FBG), $\mathrm{HbA1C}$, triglyceride $(\mathrm{TG})$, high-density lipoprotein $(\mathrm{HDLC})$, alanine aminotransferase (ALT), aspartate aminotransferase (AST), urea nitrogen (BUN), creatinine (CR), FT3, FT4, and TSH were significantly different between the non-NAFLD group and NAFLD group. Age, BMI, waist circumference, DBP, fFBG, HbA1c, total cholesterol (TC), low-density lipoprotein (LDLC), AST, and UA were all independent risk factors for NAFLD. In the normal uric acid group, variables other than SBP and TSH were independent factors of NAFLD. In the hyperuricemia group, all variables except SBP, FT4, and TSH were independent factors of NAFLD.

Conclusion: The level of uric acid is related to the occurrence of NAFLD. Hyperuricemia is one of the independent risk factors of NAFLD. TSH level is not related to the occurrence of NAFLD, while FT3 and FT4 may be related to NAFLD.

Keywords: Nonalcoholic fatty liver disease, Uric acid, TSH, FT3, FT4

\section{Introduction}

Nonalcoholic fatty liver disease (NAFLD) is a common chronic disease characterized by the accumulation of fat in the liver, which can be associated with obesity, and can progress to fibrosis, cirrhosis, and even liver cancer [1]. NAFLD is considered as an important feature of metabolic syndrome and an important factor causing cardiovascular diseases. More studies have suggested that NAFLD is related to male sexual dysfunction and reproductive

\footnotetext{
* Correspondence: chaoguanqun@zju.edu.cn

Department of General Practice, Sir Run Run Shaw Hospital, Zhejiang University, Hangzhou 310016, China
}

dysfunction [2]. NAFLD has also been shown to be associated with poor metabolism and oxidative stress [3]. Other studies have linked NAFLD to sedentary behavior and poor eating habits [4]. NAFLD is one of the most common chronic liver diseases in developed countries [5]. Except for lifestyle intervention, no drugs are currently approved for the treatment of NAFLD, so more clinical studies are needed to find new methods for the treatment and management of NAFLD.

Uric acid is mostly metabolized by the kidneys and is a product of purine metabolism in the human body. Hyperuricemia is a common manifestation in patients with chronic kidney disease, which is believed to be related to

\section{$\triangle B M C$}

(c) The Author(s). 2021 Open Access This article is licensed under a Creative Commons Attribution 4.0 International License, which permits use, sharing, adaptation, distribution and reproduction in any medium or format, as long as you give appropriate credit to the original author(s) and the source, provide a link to the Creative Commons licence, and indicate if changes were made. The images or other third party material in this article are included in the article's Creative Commons. licence, unless indicated otherwise in a credit line to the material. If material is not included in the article's Creative Commons licence and your intended use is not permitted by statutory regulation or exceeds the permitted use, you will need to obtain permission directly from the copyright holder. To view a copy of this licence, visit http://creativecommons.org/licenses/by/4.0/ The Creative Commons Public Domain Dedication waiver (http://creativecommons.org/publicdomain/zero/1.0/) applies to the data made available in this article, unless otherwise stated in a credit line to the data. 
vascular smooth muscle proliferation, endothelial dysfunction, and interstitial inflammatory infiltration [6]. Studies have shown that hyperuricemia is associated with hypertension, hyperlipidemia, diabetes, and obesity [7]. Hyperuricemia and NAFLD have similar clinical and pathological characteristics, so we speculate that there is a certain relationship between them.

Thyroid hormones T3 and T4 are synthesized in the thyroid gland and are associated with thyroglobulin [8]. Some researchers proposed that TSH, FT3, and BMI presented a nonlinear positive quadratic relationship, while FT4 and BMI presented a negative nonlinear relationship [9]. It has also been found that obese children can significantly improve the structure and function of the thyroid gland after weight loss [10]. There is also a study suggesting that thyroid function is related to cardiovascular events. Compared with those with normal thyroid function, the incidence and mortality of cardiovascular events in those with abnormal thyroid function are significantly increased [11]. Thus, thyroid function is associated with obesity and cardiovascular events. We hypothesized that thyroid hormones might also be associated with NAFLD and hyperuricemia, given their commonalities with metabolic and cardiovascular events associated with NAFLD and hyperuricemia. This study aims to further explain the correlation among NAFLD, hyperuricemia, and thyroid function by collecting data of physical examination population and using statistical analysis method and to find independent risk factors for each other.

\section{Methods}

\section{Data and methods}

Data were obtained from subjects who underwent health examination in the Health Promotion Centre of Sir Run Run Shaw Hospital of Zhejiang University from January 2017 to February 2019. The diagnosis of NAFLD is based on the following three criteria: nonalcoholic, fatty degeneration detected by imaging or histological examination, and other liver diseases excluded [12]. Exclusion subjects: (1) patients with severe cardiac, hepatic and renal insufficiency; (2) recent use of drugs affecting thyroid secretion or previous clear diagnosis of thyroid diseases or use of drugs affecting purine metabolism; (3) liver damage, alcoholism, viral liver disease, alcoholic liver disease, autoimmune liver disease, genetic, and drug-induced liver disease caused by other factors; and (4) malignant tumors and rheumatic diseases. Sir Run Run Shaw Hospital ethics committee reviewed and approved the study.

\section{Grouping}

NAFLD was consistent with no history of alcohol consumption or alcohol consumption equivalent to ethanol
$<140 \mathrm{~g} /$ week (male), ethanol $<70 \mathrm{~g} /$ week (female), except for viral hepatitis, drug-induced liver disease, total parenteral nutrition, hepatolenticular degeneration, autoimmune liver disease, and other specific diseases that can lead to fatty liver disease. According to the diagnostic criteria and exclusion criteria of NAFLD, we divided the samples into NAFLD group and non-NAFL D group.

The results were grouped according to serum uric acid levels. Serum uric acid (SUA) $>360 \mathrm{mmol} / \mathrm{L}$ (female) and SUA $>420 \mathrm{mmol} / \mathrm{L}$ (male) were enrolled in the hyperuricemia group, while the rest were enrolled in the non-hyperuricemia group.

\section{Statistical processing}

$\mathrm{R}$ software was used for statistical analysis. The measurement data conforming to the normal distribution were expressed as the mean \pm standard deviation $(-X \pm S D)$. The comparison between the two groups was conducted by $t$ test, and $P<0.05$ was considered statistically significant. Classification data were compared by chi-square test, and the difference was statistically significant by $P$ $<0.001$. The measurement data of non-normal distribution were expressed by the median (interquartile distance), the Mann-Whitney $U$ rank sum test was used for pantwise comparison, and the multivariate logistic regression analysis was used for risk factor analysis.

\section{Results}

\section{General data and correlation analysis}

Finally, 55,449 subjects were included in the analysis. $34.27 \%$ of patients were classified as NAFLD group ( $N=$ 19004 ), and $65.73 \%$ of patients were classified as nonNAFLD group $(N=36445)$. Gender ratio, age, BMI, waist circumference, systolic blood pressure, diastolic blood pressure, fasting blood glucose, HbA1c, triglyceride, high-density lipoprotein, glutamate transaminase, glutamate transaminase, urea nitrogen, creatinine, FT3, FT4, and TSH were significantly different between the two groups $(P<0.05)$ (see Table 1$)$.

\section{Risk factors for NAFLD group}

In order to better determine the correlation between the level of relevant indicators and NAFLD, we conducted logistics regression analysis. In the single-factor regression model, we divided the subjects into two categories, including gender, age, BMI, waist circumference, blood pressure, rapid blood glucose, blood lipid, liver function, uric acid, thyroid hormone, and other relevant indicators for analysis. The results showed that male, age, BMI, waist circumference, diastolic blood pressure, rapid blood glucose, HbA1c, total cholesterol, low-density lipoprotein, AST, uric acid, and FT3 were independent risk factors for NAFLD $(\mathrm{OR}>1)$. In the multivariate 
Table 1 Baseline characteristics of subjects stratified according to the Non-NAFLD or NAFLD (mean \pm SD)

\begin{tabular}{|c|c|c|c|c|}
\hline & Non-NAFLD group $(n=36445)$ & NAFLD group ( $n=19004)$ & Overall $(n=55449)$ & ${ }^{\text {a } P \text { value }}$ \\
\hline Male gender, \% & $16,731(45.9 \%)$ & $14,587(76.8 \%)$ & $31,318(56.5 \%)$ & $<0.001$ \\
\hline Age, years & $46.0 \pm 10.7$ & $48.8 \pm 9.79$ & $47.0 \pm 10.5$ & $<0.001$ \\
\hline $\mathrm{BMl}, \mathrm{kg} / \mathrm{m}^{2}$ & $22.9 \pm 2.84$ & $26.1 \pm 3.06$ & $24.0 \pm 3.28$ & $<0.001$ \\
\hline \multicolumn{5}{|l|}{ Blood pressure, $\mathrm{mmHg}$} \\
\hline Systolic blood pressure & $119 \pm 16.2$ & $128 \pm 15.2$ & $122 \pm 16.5$ & $<0.001$ \\
\hline Diastolic blood pressure & $70.8 \pm 10.9$ & $77.8 \pm 10.9$ & $73.2 \pm 11.3$ & $<0.001$ \\
\hline Waist circumference, $\mathrm{cm}$ & $79.6 \pm 8.95$ & $91.6 \pm 8.23$ & $83.7 \pm 10.4$ & $<0.001$ \\
\hline $\mathrm{FBG}, \mathrm{mmol} / \mathrm{L}$ & $5.12 \pm 0.885$ & $5.75 \pm 1.51$ & $5.34 \pm 1.18$ & $<0.001$ \\
\hline Hbalc, \% & $5.29 \pm 0.581$ & $5.67 \pm 0.923$ & $5.42 \pm 0.739$ & $<0.001$ \\
\hline Triglycerides, mg/dL & $1.37 \pm 1.03$ & $2.44 \pm 1.93$ & $1.74 \pm 1.50$ & $<0.001$ \\
\hline Total cholesterol, mg/dL & $4.72 \pm 0.909$ & $5.02 \pm 0.996$ & $4.82 \pm 0.951$ & $<0.001$ \\
\hline High density lipoprotein, mg/dL & $1.28 \pm 0.314$ & $1.07 \pm 0.242$ & $1.21 \pm 0.309$ & $<0.001$ \\
\hline Low density lipoprotein, mg/dL & $2.67 \pm 0.733$ & $2.84 \pm 0.807$ & $2.73 \pm 0.763$ & $<0.001$ \\
\hline Alanine aminotransferase, IU/L & $20.3 \pm 20.1$ & $35.2 \pm 27.2$ & $25.4 \pm 23.9$ & $<0.001$ \\
\hline Aspartate aminotransferase, IU/L & $20.1 \pm 11.1$ & $24.7 \pm 13.6$ & $21.7 \pm 12.2$ & $<0.001$ \\
\hline Uric acid, $\mu \mathrm{mol} / \mathrm{L}$ & $324 \pm 82.4$ & $397 \pm 86.1$ & $349 \pm 90.6$ & $<0.001$ \\
\hline $\mathrm{TSH}, \mathrm{mIU} / \mathrm{L}$ & $1.88 \pm 1.67$ & $1.85 \pm 1.55$ & $1.87 \pm 1.63$ & 0.034 \\
\hline $\mathrm{FT} 3, \mathrm{pg} / \mathrm{ml}$ & $2.59 \pm 0.895$ & $2.68 \pm 0.839$ & $2.62 \pm 0.877$ & $<0.001$ \\
\hline $\mathrm{FT} 4, \mathrm{ng} / \mathrm{dl}$ & $2.18 \pm 2.00$ & $2.10 \pm 1.92$ & $2.15 \pm 1.97$ & $<0.001$ \\
\hline
\end{tabular}

Note: $B P$ blood pressure, SBP systolic blood pressure, DBP diastolic blood pressure, WC waist circumference, $F B G$ fast blood glucose, SUA serum uric acid, $T C$ total cholesterol, $T G$ triglycerides, $L D L$ low-density lipoprotein, $H D L$ high-density lipoprotein, $A L T$ alanine aminotransferase, $A S T$ aspartate aminotransferase, $B U N$ blood urea nitrogen, NAFLD nonalcoholic fatty liver disease. ${ }^{a}$ Two-sided $P$ values for the difference between the non-NAFLD groups and NAFLD groups

logistic regression model, age, BMI, waist circumference, diastolic blood pressure, rapid blood glucose, HbA1c, total cholesterol, low-density lipoprotein, AST, and uric acid were all independent risk factors for NAFLD (OR $>1)$. Systolic blood pressure and TSH are independent factors (see Table 2).

\section{Risk factors for NAFLD group between hyperuricemia group and normal uric acid group}

In subjects with the normal uric acid $(N=39183)$, the NAFLD group $(N=10615)$ compared with the nonNAFLD group $(N=28568)$, sex, age, BMI, waist circumference, systolic blood pressure, diastolic blood pressure, triglyceride, total cholesterol, high-density lipoprotein cholesterol (HDL-c), low-density lipoprotein cholesterol (LDL-c), ALT, AST, FT3, FT4, etc., all have significant difference and with no difference of TSH in the two groups $(p=0.309)$. In subjects with the hyperuricemia $(N=16266)$, the fatty liver group $(N=8389)$ compared with the non-NAFLD group $(N=7877)$, sex ratio, age, BMI, waist circumference, systolic pressure, diastolic blood pressure, triglyceride, total cholesterol, HDL-c, LDL-c, ALT, AST, FT3, etc., all have significant difference and with no difference of TSH $(p=0.784)$ and FT4 $(p=0.173)$ in the two groups (see Table 3$)$. Logistic multivariate regression analysis was performed in the
Table 2 Odds ratio of NAFLD incidence by demographic and metabolic factors

\begin{tabular}{|c|c|c|c|c|}
\hline \multirow[t]{2}{*}{ Exposure } & \multicolumn{2}{|l|}{ Univariate } & \multicolumn{2}{|l|}{ Multivariate } \\
\hline & OR $(95 \% \mathrm{Cl})$ & $p$ value & OR $(95 \% \mathrm{Cl})$ & $p$ value \\
\hline Age & 1.02 & 11 & $.006-1.012)$ & $<0.001$ \\
\hline$\pi$ & $443-1.465)$ & 0.001 & 1.122) & $<0.0$ \\
\hline$P$ & $1.039(1.038-1.040)$ & $<0.001$ & $1.000(0.998-1.002)$ & 0.989 \\
\hline $\mathrm{BP}$ & 59-1.062) & $<0.001$ & .019) & $<0.001$ \\
\hline /C & 1175 & $<00$ & 7) & $<0.001$ \\
\hline 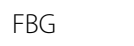 & 1.80 & $<0.001$ & 1.1 & $<0$ \\
\hline bA $1 \mathrm{c}$ & $2.235(2.167-2.307)$ & $<0.001$ & $1.295(1.230-1.365)$ & $<0.001$ \\
\hline C & $1.392(1.366-1.419)$ & $<0.001$ & $0.716(0.664-0.773)$ & $<0.001$ \\
\hline dl-c & $0.054(0.050-0.058)$ & $<0.001$ & 0.493 & $<0.001$ \\
\hline Ldl-c & )) & $<$ & 2) & $<0$ \\
\hline ST & $1.051(1.049-1.054)$ & $<0.001$ & $0.968(0.964-0.973)$ & $<0.001$ \\
\hline UA & 1.010 & $<0.001$ & .004) & $<0.0$ \\
\hline TSH & 0.988 (0.976-0.999) & 0.039 & $0.994(0.980-1.009)$ & 0.669 \\
\hline 2 & $1.122(1.099-1.146)$ & $<0.001$ & $0.941(0.899-0.983)$ & $<0.001$ \\
\hline T4 & $0.979(0.971-0.988)$ & $<0.001$ & $0.952(0.934-0.971)$ & $<0.001$ \\
\hline
\end{tabular}

Note: $B P$ blood pressure, $S B P$ systolic blood pressure, $D B P$ diastolic blood pressure, WC waist circumference, $F B G$ fast blood glucose, SUA serum uric acid, $T C$ total cholesterol, $L D L$ low-density lipoprotein, $H D L$ high-density lipoprotein, AST aspartate aminotransferase, BUN blood urea nitrogen, NAFLD nonalcoholic fatty liver disease 
Table 3 Odds ratio of NAFLD incidence in normal uric acid group and hyperuricemia group

\begin{tabular}{|c|c|c|c|c|c|c|}
\hline & \multicolumn{3}{|l|}{ Normal uric acid } & \multicolumn{3}{|l|}{ Hyperuricemia } \\
\hline & Non-NAFLD ( $N=28568)$ & NAFLD $(N=10615)$ & $p$ value & Non-NAFLD $(N=7877)$ & NAFLD $(N=8389)$ & $p$ value \\
\hline Male gender & 11,025 (38.6\%) & $6947(65.4 \%)$ & $<0.001$ & 5706 (72.4\%) & $7640(91.1 \%)$ & $<0.001$ \\
\hline Age & $46.0 \pm 10.6$ & $50.0 \pm 9.49$ & $<0.001$ & $46.2 \pm 11.2$ & $47.4 \pm 9.98$ & $<0.001$ \\
\hline BMl & $22.7 \pm 2.81$ & $25.8 \pm 2.97$ & $<0.001$ & $23.6 \pm 2.82$ & $26.4 \pm 3.12$ & $<0.001$ \\
\hline SBP & $118 \pm 16.2$ & $128 \pm 15.6$ & $<0.001$ & $122 \pm 15.8$ & $129 \pm 14.7$ & $<0.001$ \\
\hline DBP & $70.2 \pm 10.7$ & $76.8 \pm 10.8$ & $<0.001$ & $73.2 \pm 11.0$ & $79.0 \pm 10.8$ & $<0.001$ \\
\hline WC & $78.5 \pm 8.72$ & $90.2 \pm 8.30$ & $<0.001$ & $83.3 \pm 8.78$ & $93.3 \pm 7.80$ & $<0.001$ \\
\hline FBG & $5.11 \pm 0.896$ & $5.85 \pm 1.72$ & $<0.001$ & $5.17 \pm 0.841$ & $5.62 \pm 1.17$ & $<0.001$ \\
\hline $\mathrm{HbA1c}$ & $5.29 \pm 0.588$ & $5.75 \pm 1.02$ & $<0.001$ & $5.31 \pm 0.556$ & $5.57 \pm 0.775$ & $<0.001$ \\
\hline TG & $1.28 \pm 0.898$ & $2.21 \pm 1.74$ & $<0.001$ & $1.69 \pm 1.37$ & $2.72 \pm 2.12$ & $<0.001$ \\
\hline $\mathrm{TC}$ & $4.70 \pm 0.903$ & $4.98 \pm 0.997$ & $<0.001$ & $4.79 \pm 0.929$ & $5.06 \pm 0.994$ & $<0.001$ \\
\hline $\mathrm{Hdll}-\mathrm{c}$ & $1.31 \pm 0.313$ & $1.09 \pm 0.250$ & $<0.001$ & $1.20 \pm 0.300$ & $1.03 \pm 0.227$ & $<0.001$ \\
\hline Ldl-c & $2.66 \pm 0.727$ & $2.85 \pm 0.804$ & $<0.001$ & $2.72 \pm 0.754$ & $2.83 \pm 0.811$ & $<0.001$ \\
\hline ALT & $19.4 \pm 20.6$ & $32.1 \pm 26.8$ & $<0.001$ & $23.3 \pm 17.9$ & $39.2 \pm 27.2$ & $<0.001$ \\
\hline AST & $19.8 \pm 11.3$ & $23.6 \pm 13.5$ & $<0.001$ & $21.3 \pm 10.2$ & $26.1 \pm 13.6$ & $<0.001$ \\
\hline TSH & $1.89 \pm 1.58$ & $1.87 \pm 1.70$ & 0.309 & $1.82 \pm 1.94$ & $1.81 \pm 1.34$ & 0.748 \\
\hline FT3 & $2.58 \pm 0.892$ & $2.68 \pm 0.830$ & $<0.001$ & $2.62 \pm 0.905$ & $2.67 \pm 0.849$ & $<0.001$ \\
\hline FT4 & $2.17 \pm 2.00$ & $2.06 \pm 1.89$ & $<0.001$ & $2.18 \pm 2.00$ & $2.14 \pm 1.95$ & 0.173 \\
\hline
\end{tabular}

two groups respectively. In the normal uric acid group, variables other than systolic blood pressure and TSH were independent factors of NAFLD. In the hyperuricemia group, all variables except systolic blood pressure, FT4, and TSH were independent factors of NAFLD (see Table 4).

Table 4 Odds ratio of NAFLD incidence in normal uric acid group and hyperuricemia group

\begin{tabular}{llllll}
\hline Exposure & Normal uric acid & & & Hyperuricemia & \\
\cline { 2 - 3 } \cline { 6 - 7 } & OR $(95 \% \mathrm{Cl})$ & $\boldsymbol{p}$ value & & OR $(95 \% \mathrm{Cl})$ & $\boldsymbol{p}$ value \\
\hline Age & $1.009(1.005-1.012)$ & $<0.001$ & $1.008(1.004-1.012)$ & $<0.001$ \\
BMI & $1.117(1.102-1.132)$ & $<0.001$ & $1.108(1.088-1.128)$ & $<0.001$ \\
SBP & $1.002(1.000-1.005)$ & 0.103 & $0.996(0.992-1.000)$ & 0.047 \\
DBP & $1.014(1.010-1.018)$ & $<0.001$ & $1.021(1.015-1.026)$ & $<0.001$ \\
WC & $1.009(1.103-1.114)$ & $<0.001$ & & $1.100(1.092-1.108)$ & $<0.001$ \\
FBG & $1.086(1.044-1.130)$ & $<0.001$ & & $1.173(1.103-1.248)$ & $<0.001$ \\
HbA1C & $1.285(1.206-1.370)$ & $<0.001$ & & $1.231(1.125-1.348)$ & $<0.001$ \\
TG & $1.602(1.528-1.681)$ & $<0.001$ & & $1.376(1.304-1.454)$ & $<0.001$ \\
TC & $0.703(0.639-0.734)$ & $<0.001$ & & $0.734(0.647-0.833)$ & $<0.001$ \\
Hdl-C & $0.483(0.413-0.564)$ & $<0.001$ & & $0.490(0.396-0.607)$ & $<0.001$ \\
Ldl-C & $1.825(1.643-2.027)$ & $<0.001$ & & $1.660(1.443-1.910)$ & $<0.001$ \\
ALT & $1.033(1.030-1.037)$ & $<0.001$ & & $1.040(1.036-1.044)$ & $<0.001$ \\
AST & $0.966(0.960-0.971)$ & $<0.001$ & & $0.975(0.968-0.982)$ & $<0.001$ \\
TSH & $1.004(0.986-1.022)$ & 0.659 & $0.983(0.960-1.007)$ & 0.158 \\
FT3 & $0.926(0.875-0.978)$ & $<0.001$ & $0.927(0.856-1.000)$ & 0.056 \\
FT4 & $0.950(0.927-0.973)$ & $<0.001$ & $0.945(0.912-0.977)$ & $<0.001$ \\
\hline
\end{tabular}

Analysis of the collinearity of TSH factors

As mentioned above, TSH was not significantly associated with NAFLD in multivariate logistic regression. In order to explore the factors between TSH and NAFLD, we found that after adjusting for age and gender, TSH was associated with NAFLD when other variables were adjusted separately; when TSH was adjusted together with BMI, waist circumference and TG, TSH was not associated with NAFLD (see Table 5).

\section{Discussion}

As a common chronic liver disease, NAFLD is on the rise in Asia and is closely associated with liver cancer and mortality [13]. Due to the increasing incidence of obesity and metabolic syndrome, the incidence of NAFLD is also on the rise, leading to cirrhosis and liver cancer [14]. Therefore, NAFLD has become a major global health problem. The investigators [15] noted that NAFLD was associated with components of the metabolic syndrome, including type 2 diabetes, obesity, hypertension, and dyslipidemia. This is consistent with our findings. It is also pointed out that NAFLD is not only related to genetic variation, but also to environmental factors. Our results showed that in addition to blood lipid, blood pressure, and blood sugar, NAFLD was also associated with gender, liver function, thyroid function, uric acid, and other indicators, and the comparison between the two groups had statistical significance. In the past decade, there have been 
Table 5 Logistic regression for NAFLD outcome with individual risk factors

\begin{tabular}{lll}
\hline Adjusted TSH & OR $(95 \%$ Cl) & $P$ value \\
\hline Gender and age & $1.029(1.017-1.041)$ & $<0.001$ \\
BMI & $1.011(0.999-1.025)$ & 0.080 \\
SBP & $1.022(1.010-1.032)$ & $<0.001$ \\
DBP & $1.020(1.008-1.062)$ & $<0.001$ \\
WC & $1.009(0.995-1.023)$ & 0.199 \\
FBG & $1.032(1.019-1.044)$ & $<0.001$ \\
HbA1C & $1.028(1.016-1.041)$ & $<0.001$ \\
TG & $1.009(0.997-1.021)$ & 0.15 \\
TC & $1.019(1.007-1.031)$ & 0.002 \\
Hdl-c & $1.034(1.021-1.046)$ & $<0.001$ \\
Ldl-c & $1.025(1.014-1.037)$ & $<0.001$ \\
ALT & $1.016(1.003-1.029)$ & 0.008 \\
AST & $1.019(1.007-1.031)$ & 0.002 \\
UA & $1.017(1.005-1.029)$ & 0.007 \\
FT3 & $1.029(1.017-1.041)$ & $<0.001$ \\
FT4 & $1.028(1.017-1.041)$ & $<0.001$ \\
\hline
\end{tabular}

many studies confirming the correlation between uric acid level and metabolic syndrome [16].

It has also been pointed out that uric acid level is an important factor causing metabolic syndrome [7]. In our study, multivariate regression analysis concluded that increased uric acid level was one of the risk factors for NAFLD. This conclusion is consistent with previous studies [17]. Considering that hyperuricemia is a risk factor for fatty liver disease, we consider the following reasons: (1) uric acid is an oxidant that can cause oxidative stress on the liver, thus promoting the development of NAFLD [18]; (2) uric acid may be released by necrotic cells, leading to sterile inflammation, and NAFLD may produce necrotic hepatocytes, which may also be the reason why uric acid is associated with NAFLD [19]; and (3) both hyperuricemia and NAFLD are related to metabolic syndrome, and the reason for the increase of uric acid in metabolic syndrome is that hyperinsulinemia leads to the reduction of uric acid exclusion, so it is speculated that the necessary relationship between uric acid level and NAFLD.

Our study showed that age, BMI, waist circumference, diastolic blood pressure, rapid blood glucose, HbA1c, total cholesterol, low-density lipoprotein, AST, and uric acid were all independent risk factors for NAFLD $(\mathrm{OR}>1)$. Male, total cholesterol, highdensity lipoprotein, AST, FT3, and FT4 were protective factors $(\mathrm{OR}<1)$. At the same time, we divided the NAFLD group and the control group according to the uric acid level. In the normal uric acid group, variables other than systolic blood pressure and TSH were independent factors of NAFLD. In the hyperuricemia group, all variables except systolic blood pressure, FT4, and TSH were independent factors of NAFLD.

We found that FT3 and FT4 were considered as protective factors of NAFLD. After secondary grouping, FT3 and FT4 were protective factors for NAFLD under normal uric acid conditions. In the case of increased uric acid, only FT4 was a protective factor for NAFLD. Thyroid hormone has a metabolic effect on protein, carbohydrate, and fat. The decrease of thyroid level is related to the low metabolism, which can be manifested as weight gain, increased blood lipid, and increased blood sugar [20]. Studies have found that thyroxine is related to blood lipids and blood sugar, which can lead to obesity, hyperlipidemia, insulin resistance and other components of metabolic syndrome [21]. One study indicated that the FT3/FT4 ratio was a risk factor for NAFLD, while there was no direct correlation between reduced thyroid function and NAFLD [22]. However, another study confirmed the association between subclinical hypothyroidism and clinical hypothyroidism and NAFLD [23]. Similarly, in a retrospective study, subclinical hypothyroidism was not associated with an increased incidence of NAFLD [24]. In view of the controversial results, the researchers collected the literature and conducted a meta-analysis, concluding that subclinical hypothyroidism was not associated with NAFL $\mathrm{D}$, and thyroid hormone levels were not associated with the presence or absence of NAFLD [25]. We found that TSH was not associated with NAFLD regardless of uric acid levels, consistent with the results of the metaanalysis. However, it was found unexpectedly that FT3 and FT4 were associated with NAFLD, and there were still differences after secondary grouping according to uric acid levels, which were considered as factors. However, the trend of the two levels is opposite, so further prospective studies are needed to further confirm the correlation between thyroid hormone and NAFLD. In addition, thyroid hormone levels are also affected by a variety of factors, including stress, so the results are unstable and controversial.

Since our study found that there was no correlation between TSH and NAFLD, which was inconsistent with other studies, we hoped to look for common clues. We found that after adjusting for age and gender, TSH was associated with NAFLD when other variables were adjusted separately; when TSH was adjusted together with BMI, waist circumference, and TG, TSH was not associated with NAFLD. Therefore, we hypothesized that the correlation between TSH and NAFLD may be affected by body weight and blood lipids, which needs to be further confirmed by further studies. In summary, NAFLD, hyperuricemia, 
and thyroid dysfunction are all related to components of metabolic syndrome, and hyperuricemia is a risk factor for the increased incidence of NAFLD. Whether uric acid level is normal or not, TSH is not related to NAFLD, while FT3 and FT4 are related to NAFLD, but whether they are the factors of NAFLD remains to be confirmed.

\section{Conclusion}

The level of uric acid is related to the occurrence of NAFLD. Hyperuricemia is one of the independent risk factors of NAFLD. TSH level is not related to the occurrence of NAFLD, while FT3 and FT4 may be related to NAFLD.

\section{Abbreviations}

NAFLD: Nonalcoholic fatty liver disease; SUA: Serum uric acid; HDL-c: Highdensity lipoprotein cholesterol; LDL-c: Low-density lipoprotein cholesterol

\section{Acknowledgements}

Not applicable

\section{Authors' contributions}

Guanqun Chao wrote the article and analyzed. Liying Chen guided the study. The authors read and approved the final manuscript.

\section{Funding}

This research was supported by funding from National Natural Science Foundation of China (81973598,81573760); Zhejiang Provincial Natural Science Foundation of China under Grant No.LY18H030001; the Medicine and Health Science and Technology Plan Projects in Zhejiang province (2017KY413), Traditional Chinese Medicine Science and Technology Plan of Zhejiang Province (2017ZA089, 2016ZB071, 2015ZZ012, 2014ZA030); Medical Health Platform Plan Projects of Zhejiang Province (2015RCA020); Zhejiang Provincial Natural Science Foundation of China (LY16H030010).

\section{Availability of data and materials}

The datasets used and/or analyzed during the current study are available from the corresponding author on reasonable request.

\section{Declarations}

Ethics approval and consent to participate

Sir Run Run Shaw Hospital ethics committee reviewed and approved the study.

\section{Consent for publication}

Not applicable.

\section{Competing interests}

The authors declare that they have no competing interests.

Received: 14 August 2020 Accepted: 22 April 2021

Published online: 03 May 2021

\section{References}

1. Del Río-Moreno M, Alors-Pérez E, González-Rubio S, Ferrín G, Reyes O, Rodríguez-Perálvarez $\mathrm{M}$, et al. Dysregulation of the splicing machinery is associated to the development of non-alcoholic fatty liver disease. J Clin Endocrinol Metab. 2019;104(8):3389.

2. Hawksworth DJ, Burnett AL. Nonalcoholic fatty liver disease, male sexual dysfunction, and infertility: common links, common problems. Sex Med Rev. 2019;8(2):274.

3. Gheflati A, Adelnia E, Nadjarzadeh A. The clinical effects of purslane (Portulaca oleracea) seeds on metabolic profiles in patients with nonalcoholic fatty liver disease: A randomized controlled clinical trial. Phytother Res. 2019;33(5):1501-9. https://doi.org/10.1002/ptr.6342.
4. Zhou X, Li Y, Zhang X, Guan YY, Puentes Y, Zhang F, et al. Independent markers of non-alcoholic fatty liver disease in a gentrifying population based Chinese cohort. Diabetes Metab Res Rev. 2019;35:e3156.

5. Gallego-Durán R, Álvarez-Amor L, Gil-Gómez A, Rojas Á, Muñoz-Hernández R, Cádernas-García A, et al. Metabolic characterization of two different nonalcoholic fatty liver disease pre-clinical mouse models. Rev Esp Enferm Dig. 2019;111:301. https://doi.org/10.17235/reed.2019.6083/2018.

6. Fan S, Zhang P, Wang AY, Wang $X$, Wang L, Li G, et al. Hyperuricemia and its related histopathological features on renal biopsy. BMC Nephrol. 2019; 20(1):95. https://doi.org/10.1186/s12882-019-1275-4.

7. Abbasi S, Haleem N, Jadoon S, Farooq A. Association of non-alcoholic fatty liver disease with serum uric acid. J Ayub Med Coll Abbottabad. 2019;31(1): 64-6.

8. Citterio CE, Targovnik HM, Arvan P. The role of thyroglobulin in thyroid hormonogenesis. Nat Rev Endocrinol. 2019;15(6):323.

9. Song Q, Chen X, Su Y, Xie Z, Wang S, Cui B. Age and gender specific thyroid hormones and their relationships with body mass index in a large Chinese population. Int J Endocrinol Metab. 2019;17(1):e66450. https://doi. org/10.5812/ijem.66450.

10. Licenziati MR, Valerio G, Vetrani I, De Maria G, Liotta F, Radetti G. Altered thyroid function and structure in overweight and obese children and adolescents: reversal after weight loss. J Clin Endocrinol Metab. 2019;104(7): 2757.

11. Manolis AA, Manolis TA, Melita H, Manolis AS. Subclinical thyroid dysfunction and cardiovascular consequences: an alarming wake-up call? Trends Cardiovasc Med. 2019;30(2):57.

12. Hashimoto E, Taniai M, Tokushige K. Characteristics and diagnosis of NAFL D/NASH. J Gastroenterol Hepatol. 2013;28(Suppl 4):64-70.

13. Li J, Zou B, Yeo YH, Feng Y, Xie X, Lee DH, et al. Prevalence, incidence, and outcome of non-alcoholic fatty liver disease in Asia, 1999-2019: a systematic review and meta-analysis. Lancet Gastroenterol Hepatol. 2019;4(5):389.

14. Abshagen K, Mense L, Fischer F, Liebig M, Schaeper U, Navarro G, et al. Repin1 deficiency in liver tissue alleviates NAFLD progression in mice. J Adv Res. 2018:16:99-111.

15. lqbal U, Perumpail BJ, Akhtar D, Kim D, Ahmed A. The epidemiology, risk profiling and diagnostic challenges of nonalcoholic fatty liver disease. Medicines (Basel). 2019;6(1):E41.

16. Huang $\mathrm{Q}, \mathrm{Yu}$ J, Zhang X, Liu S, Ge Y. Association of the serum uric acid level with liver histology in biopsy-proven non-alcoholic fatty liver disease. Biomed Rep. 2016;5(2):188-92. https://doi.org/10.3892/br.2016.698.

17. Li Y, Xu C, Yu C, Xu L, Miao M. Association of serum uric acid level with non-alcoholic fatty liver disease: a cross-sectional study. J Hepatol. 2009; 50(5):1029-34. https://doi.org/10.1016/j.jhep.2008.11.021.

18. Baskol G, Baskol M, Kocer D. Oxidative stress and antioxidant defenses in serum of patients with non-alcoholicsteatohepatitis. Clin Biochem. 2007; 40(11):776-80. https://doi.org/10.1016/j.clinbiochem.2007.02.006.

19. Petta S, Cammà C, Cabibi D, Di Marco V, Craxì A. Hyperuricemia is associated with histological liver damage in patients with non-alcoholic fatty liver disease. Aliment Pharmacol Ther. 2011;34(7):757-66. https://doi. org/10.1111/j.1365-2036.2011.04788.x.

20. Pucci E, Chiovato L, Pinchera A. Thyroid and lipid metabolism. Int J Obes Relat Metab Disord. 2000;24(Suppl 2):S109-12. https://doi.org/10.1038/sj.ijo. 0801292.

21. Erdogan M, Canataroglu A, Ganidagli S, Kulaksızoglu M. Metabolic syndrome prevalence in subclinic and overt hypothyroid patients and the relation among metabolic syndrome parameters. J Endocrinol Investig. 2011;34(7): 488-92. https://doi.org/10.3275/7202.

22. Gökmen FY, Ahbab S, Ataoğlu HE, Türker BÇ, Çetin F, Türker F, et al. FT3/FT4 ratio predicts non-alcoholic fatty liver disease independent of metabolic parameters in patients with euthyroidism and hypothyroidism. Clinics (Sao Paulo). 2016;71(4):221-5. https://doi.org/10.6061/clinics/2016(04)08.

23. Ludwig U, Holzner D, Denzer C, Greinert A, Haenle MM, Oeztuerk S, et al. Subclinical and clinical hypothyroidism and non-alcoholic fatty liver disease: a cross-sectional study of a random population sample aged 18 to 65 years. BMC Endocr Disord. 2015;15(1):41. https://doi.org/10.11 86/s12902-015-0030-5.

24. Lee KW, Bang KB, Rhee EJ, Kwon HJ, Lee MY, Cho YK. Impact of hypothyroidism on the development of non-alcoholic fatty liver disease: a 4-yearretrospective cohort study. Clin Mol Hepatol. 2015;21(4):372-8. https:// doi.org/10.3350/cmh.2015.21.4.372 
25. Jaruvongvanich V, Sanguankeo A, Upala S. Nonalcoholic fatty liver disease is not associated with thyroid hormone levels and hypothyroidism: a systematic review and meta-analysis. Eur Thyroid J. 2017;6(4):208-15. https://doi.org/10.1159/000454920.

\section{Publisher's Note}

Springer Nature remains neutral with regard to jurisdictional claims in published maps and institutional affiliations.

Ready to submit your research? Choose BMC and benefit from:

- fast, convenient online submission

- thorough peer review by experienced researchers in your field

- rapid publication on acceptance

- support for research data, including large and complex data types

- gold Open Access which fosters wider collaboration and increased citations

- maximum visibility for your research: over $100 \mathrm{M}$ website views per year

At $\mathrm{BMC}$, research is always in progress. 